\title{
The Boundedness of Marcinkiewicz Integrals Associated with Schrödinger Operator on Morrey Spaces
}

\section{Dongxiang Chen and Fangting Jin}

Jiangxi Normal University, Nanchang 330022, China

Correspondence should be addressed to Dongxiang Chen; chendx020@yahoo.com.cn

Received 22 April 2014; Accepted 18 July 2014; Published 20 August 2014

Academic Editor: Dachun Yang

Copyright (C) 2014 D. Chen and F. Jin. This is an open access article distributed under the Creative Commons Attribution License, which permits unrestricted use, distribution, and reproduction in any medium, provided the original work is properly cited.

Let $L=-\Delta+V$ be a Schrödinger operator, where $V$ belongs to some reverse Hölder class. The authors establish the boundedness of Marcinkiewicz integrals associated with Schrödinger operators and their commutators on Morrey spaces.

\section{Introduction}

In this paper, we consider the Schrödinger operator

$$
L=-\Delta+V(x)
$$

in $R^{d}, d \geq 3$, where $V(x)$ is a nonnegative potential belonging to the reverse Hölder class $\mathrm{RH}_{q}$ for some exponent $q>d / 2$.

A nonnegative locally $L^{q}$ integrable function $V(x)$ on $R^{d}$ is said to belong to $\mathrm{RH}_{q}(q>1)$ if there exists $C>0$ such that the reverse Hölder inequality,

$$
\left(\frac{1}{|B|} \int_{B} V(y)^{q} d y\right)^{1 / q} \leq \frac{C}{|B|} \int_{B} V(y) d y,
$$

holds true for every ball $B \subset R^{d}$. We introduce the definition of the reverse Hölder index of $V$ as $q_{0}=\sup \left\{q: V \in \mathrm{RH}_{q}\right\}$. It is known that $V \in \mathrm{RH}_{q}$ implies $V \in \mathrm{RH}_{q+\epsilon}$ for some $\epsilon>0$.

The Marcinkiewicz integral operator $\mu$ is defined by

$$
\mu f(x)=\left(\int_{0}^{\infty}\left|\int_{|x-y| \leq t} \frac{\Omega(x-y)}{|x-y|^{d-1}} f(y) d y\right|^{2} \frac{d t}{t^{3}}\right)^{1 / 2} .
$$

The above operator was introduced by Stein in [1] as an extension of the notion of Marcinkiewicz function from one dimension to higher dimensions.
Similar to the classical Marcinkiewicz function $\mu$, we define the Marcinkiewicz function $\mu_{j}^{L}$ associated with the Schrödinger operator $L$ by

$$
\mu_{j}^{L} f(x)=\left(\int_{0}^{\infty}\left|\int_{|x-y| \leq t} K_{j}^{L}(x, y) f(y) d y\right|^{2} \frac{d t}{t^{3}}\right)^{1 / 2},
$$

where $K_{j}^{L}(x, y)=\widetilde{K}_{j}^{L}(x, y)|x-y|$ and $\widetilde{K}_{j}^{L}(x, y)$ is the kernel of $R_{j}^{L}=\left(\partial / \partial x_{j}\right) L^{-1 / 2}, j=1, \ldots, d$. In particular, when $V=0$, $K_{j}^{\Delta}(x, y)=\widetilde{K_{j}^{\Delta}}(x, y)|x-y|=\left(\left(x_{j}-y_{j}\right) /|x-y|\right) /|x-y|^{d-1}$ and $\widetilde{K_{j}^{\Delta}}(x, y)$ is the kernel of $R_{j}=\left(\partial / \partial x_{j}\right) \Delta^{-1 / 2}, j=1, \ldots, d$. So, $\mu_{j}^{\Delta}$ is defined by

$$
\mu_{j}^{\Delta} f(x)=\left(\int_{0}^{\infty}\left|\int_{|x-y| \leq t} K_{j}^{\Delta}(x, y) f(y) d y\right|^{2} \frac{d t}{t^{3}}\right)^{1 / 2} .
$$

Lemma 1 (see [2]). For any $l>0$, there exists $C_{l}>0$ such that

$$
\begin{gathered}
\left|K_{j}^{L}(x, y)\right| \leq \frac{C_{l}}{(1+|x-y| / \rho(y))^{l}} \frac{1}{|x-y|^{d-1}}, \\
\left|K_{j}^{L}(x, y)-K_{j}(x, y)\right| \leq C \frac{\rho(x)}{|x-y|^{d-2}},
\end{gathered}
$$

where $\rho$ is the auxiliary function as follows. 
Gao and Tang [2] have shown that Marcinkiewicz integral $\mu_{j}^{L}$ is bounded on $L^{p}\left(R^{d}\right)$ for $1<p<\infty$ and bounded from $L^{1}\left(R^{d}\right)$ to weak $L^{1}\left(R^{d}\right)$. Meanwhile they also proved $\mu_{j}^{L}$ are bounded on $\mathrm{BMO}_{L}\left(R^{d}\right)$ and also mapped from $H_{L}^{1}\left(R^{d}\right)$ to $L^{1}\left(R^{d}\right)$ under the assumption that $K_{j}^{L}$ satisfy the condition in Lemma 1.

When $K_{j}^{L}$ satisfies the estimates in Lemma 8 in Section 2 below, Chen and Zou [3] also proved that the Marcinkiewicz integral $\mu_{j}^{L}$ has the same boundedness.

Now we give the definition of the commutator generalized by $\mu_{j}^{L}$ and $b$ by

$$
\begin{aligned}
& {\left[b, \mu_{j}^{L}\right](f)(x)} \\
& =\left(\int_{0}^{\infty}\left|\int_{|x-y| \leq t} K_{j}^{L}(x, y)(b(x)-b(y)) f(y) d y\right|^{2} \frac{d t}{t^{3}}\right)^{1 / 2},
\end{aligned}
$$

and the definition of the commutator generalized by $\mu_{j}^{\Delta}$ and $b$ by

$$
\begin{aligned}
& {\left[b, \mu_{j}^{\Delta}\right](f)(x)} \\
& =\left(\int_{0}^{\infty}\left|\int_{|x-y| \leq t} K_{j}^{\Delta}(x, y)(b(x)-b(y)) f(y) d y\right|^{2} \frac{d t}{t^{3}}\right)^{1 / 2} .
\end{aligned}
$$

Let $\rho(x)$ be the auxiliary function defined by

$$
\begin{aligned}
\rho(x) & =\frac{1}{m_{V}(x)} \\
& =\sup \left\{r>0: \frac{1}{r^{d-2}} \int_{B(x, r)} V \leq 1\right\}, \quad x \in R^{d} .
\end{aligned}
$$

Obviously, $0<m_{V}(x)<\infty$ if $V \neq 0$. In particular, $m_{V}(x)=1$ with $V=1$ and $m_{V}(x) \sim(1+|x|)$ with $V=|x|^{2}$.

In this paper, we write $\Psi(B)=\left(1+r m_{V}(B)\right)^{\theta}$ where $m_{V}(B)=(1 /|B|) \int_{B} m_{V}(x) d x$ and $\theta>0$, and $r$ denotes the radius of $B$.

The maximal operator $M_{V} f(x)$ is defined by

$$
M_{V} f(x)=\sup _{x \in B} \frac{1}{\Psi(B)|B|} \int_{B}|f(x)| d x .
$$

When $V=0$, we denote $M_{0} f(x)$ by $M f(x)$ (the standard Hardy-Littlewood maximal function). It is easy to see that $|f(x)| \leq M_{V} f(x) \leq M f(x)$ for a.e $x \in R^{d}$. This information can be found in [4].

Proposition 2 (see [5]). There exists $k_{0}>0$ such that

$$
\frac{1}{C}\left(1+\frac{|x-y|}{\rho(x)}\right)^{-k_{0}} \leq \frac{\rho(y)}{\rho(x)} \leq C\left(1+\frac{|x-y|}{\rho(x)}\right)^{k_{0} /\left(k_{0}+1\right)} .
$$

In particular, $\rho(x) \sim \rho(y)$ if $|x-y|<C \rho(x)$.
Proposition 3 (see [6]). There exists a sequence of points $x_{j}$, $j \geq 1$, in $R^{d}$, so that the family $Q_{j}=B\left(x_{j}, \rho\left(x_{j}\right)\right), j \geq 1$, satisfies the following.

(i) $\bigcup_{j} Q_{j}=R^{d}$.

(ii) For every $\sigma \geq 1$ there exist constants $C$ and $N_{1}$ such that $\sum_{j} \chi_{\sigma Q_{j}} \leq C \sigma^{N_{1}}$.

Tang and Dong [7] first introduced some type Morrey space associated with Schrödinger operators. Meanwhile they obtained the strong and weak boundedness of singular integral, fractional integral, and their commutators in Morrey spaces. Inspired by their work, we give another type of Morrey space, but in fact these two kinds of Morrey spaces are the same when their exponents are restricted in some exceeding. Recently, plenty of famous results on Schrödinger operators have appeared; we refer to [8-13].

We now present the definition of Morrey spaces associated with Schrödinger operators which we needed in this paper.

Definition 4. Let $f \in L_{\text {loc }}^{q}\left(R^{d}\right), 1 \leq p \leq q<+\infty$; we say $f \in M_{p}^{q}\left(R^{d}\right)$ (Morrey spaces) provided that

$$
\|f\|_{M_{p}^{q}\left(R^{d}\right)}=\sup _{B \subset R^{d}}|B|^{1 / q-1 / p}\left(\int_{B}|f(x)|^{p} d x\right)^{1 / p}<+\infty,
$$

where $B=B\left(x_{0}, r\right)$.

Obviously, when $p=q$, the space $M_{p}^{q}\left(R^{d}\right)$ is the class Lebesgue space $L^{p}\left(R^{d}\right)$.

Definition 5. Let $f \in L_{\mathrm{loc}}^{q}\left(R^{d}\right), 1 \leq p \leq q<+\infty, \alpha \in(-\infty$, $+\infty)$, and $V \in \mathrm{RH}_{q}(q>1)$; we say $f \in M_{p, V}^{q, \alpha}\left(R^{d}\right)$ (Morrey space associated with Schrödinger operators) provided that

$$
\begin{aligned}
& \|f\|_{M_{p, V}^{q, \alpha}\left(R^{d}\right)} \\
& =\sup _{B \subset R^{d}}\left(1+\frac{r}{\rho\left(x_{0}\right)}\right)^{\alpha}|B|^{1 / q-1 / p} \\
& \quad \times\left(\int_{B}|f(x)|^{p} d x\right)^{1 / p}<+\infty,
\end{aligned}
$$

where $B=B\left(x_{0}, r\right)$.

In this note we will investigate the boundedness for the commutators of Marcinkiewicz inegral associated with Schrödinger operators on Morrey spaces given in Definition 5.

Our results can be formulated as follows.

Theorem 6. Suppose $\alpha \in(-\infty,+\infty)$ and $V \in R H_{q}(q>1)$.

(i) If $1<p \leq q<+\infty$, then

$$
\left\|\mu_{j}^{L} f\right\|_{M_{p, V}^{q, \alpha}\left(R^{d}\right)} \leq C\|f\|_{M_{p, V}^{q, \alpha}\left(R^{d}\right)},
$$


(ii) If $1=p \leq q<+\infty$, then, for any $\lambda>0$,

$$
\begin{aligned}
& \lambda\left(1+\frac{r}{\rho(x)}\right)^{\alpha}\left|\left\{y \in B(x, r):\left|\mu_{j}^{L} f(y)\right|>\lambda\right\}\right| \\
& \leq C|B(x, r)|^{1-1 / q}\|f\|_{M_{p, V}^{q, \alpha}\left(R^{d}\right)}
\end{aligned}
$$

holds for all balls $B(x, r)$, where $C$ is independent of $x$, $r, \lambda$, and $f$.

For $\theta>0$, we define the class $\mathrm{BMO}_{\theta}(\rho)$ of locally integrable functions $b$ such that

$$
\frac{1}{|B(x, r)|} \int_{B(x, r)}\left|b(y)-b_{B}\right| d y \leq C\left(1+\frac{r}{\rho(x)}\right)^{\theta},
$$

for all $x \in R^{d}$ and $r>0$, where $b_{B}=(1 /|B|) \int_{B} b$. A norm for $b \in \mathrm{BMO}_{\theta}(\rho)$, denoted by $[b]_{\theta}$, is given by the infimum of the constants in the inequalities above. Notice that if we let $\theta=0$, we obtain the John-Nirenberg space BMO.

Theorem 7. Suppose $b \in \mathrm{BMO}_{\theta}(\rho), \theta>0, \alpha \in(-\infty,+\infty)$.

(i) If $1<p \leq q<+\infty$, then

$$
\left\|\left[b, \mu_{j}^{L}\right] f\right\|_{M_{p, V}^{q, \alpha}\left(R^{d}\right)} \leq C[b]_{\theta}\|f\|_{M_{p, V}^{q, \alpha}\left(R^{d}\right)}
$$

where $C$ is independent of $f$.

(ii) There exists a constant $C>0$, such that, for all $\lambda>0$,

$$
\begin{aligned}
& \lambda\left(1+\frac{r}{\rho(x)}\right)^{\alpha}\left|\left\{y \in R^{d}:\left|\left[b, \mu_{j}^{L}\right] f(y)\right|>\lambda\right\}\right| \\
& \quad \leq C|B(x, r)|^{1-1 / q} \int_{R^{d}} \frac{|f(y)|}{\lambda}\left(1+\log ^{+} \frac{|f(y)|}{\lambda}\right) d y .
\end{aligned}
$$

Throughout this paper, $C$ denotes the constants that are independent of the main parameters involved but whose value may differ from line to line. By $A \sim B$, we mean that there exists a constant $C>1$ such that $1 / C \leq A / B \leq C$.

\section{Notation and Preliminaries}

Shen [5] gave the following kernel estimate that we need.

Lemma 8. If $V \in R H_{q}(q>1)$, then one has

(i) for every $N$ there exists a constant $C$ such that

$$
\left|K_{j}^{L}(x, z)\right| \leq \frac{C(1+|x-z| / \rho(x))^{-N}}{|x-z|^{d-1}}
$$

(ii) for every $N$ and $0<\delta<\min \left\{1,1-d / q_{0}\right\}$ there exists a constant $C$ such that

$$
\left|K_{j}^{L}(x, z)-K_{j}^{L}(y, z)\right| \leq \frac{C|x-y|^{\delta}(1+|x-z| / \rho(x))^{-N}}{|x-z|^{d-1+\delta}}
$$

where $|x-y|<(2 / 3)|x-z|$, (iii) for every $0<\sigma<2-d / q_{0}$, one has

$$
\begin{gathered}
\left|K_{j}^{L}(x, z)-K_{j}^{\Delta}(x, z)\right| \leq \frac{C}{|x-z|^{d-1}}\left(\frac{|x-z|}{\rho(z)}\right)^{\sigma}, \\
\text { where } K_{j}^{\Delta}(x, z)=\left(\left(x_{j}-z_{j}\right) /|x-z|\right) /|x-z|^{d-1} .
\end{gathered}
$$

Lemma 9 (see [14]). Let $\theta>0$ and $1 \leq s<\infty$. If $b \in$ $\mathrm{BMO}_{\theta}(\rho)$, then

$$
\left(\frac{1}{|B|} \int_{B}\left|b-b_{B}\right|^{s}\right)^{1 / s} \leq C[b]_{\theta}\left(1+\frac{r}{\rho(x)}\right)^{\theta^{\prime}}
$$

for all $B=B(x, r)$, with $x \in R^{d}$ and $r>0$, where $\theta^{\prime}=\left(k_{0}+1\right) \theta$ and $k_{0}$ is the constant appearing in Proposition 2.

Corollary 10 (see [14]). Let $b \in B M O_{\theta}(\rho), B=B\left(x_{0}, r\right)$, and $s \geq 1$; then

$$
\left(\frac{1}{\left|2^{k} B\right|} \int_{2^{k} B}\left|b-b_{B}\right|^{s}\right)^{1 / s} \leq C[b]_{\theta} k\left(1+\frac{2^{k} r}{\rho\left(x_{0}\right)}\right)^{\theta^{\prime}},
$$

for all $k \in N$, with $\theta^{\prime}$ as in Lemma 9 .

From Lemma 9, the author [15] proved the JohnNirenberg inequality for $\mathrm{BMO}_{\theta}(\rho)$.

Proposition 11 (see [15]). Suppose that $f$ is in $B M O_{\theta}(\rho)$. There exist positive constants $\gamma$ and $C$ such that

$$
\sup _{B} \frac{1}{|B|} \int_{B} \exp \left\{\frac{\gamma}{[f]_{\theta} \Psi_{\theta^{\prime}}(B)}\left|f(x)-f_{B}\right|\right\} d x \leq C,
$$

where $f_{B}=(1 /|B|) \int_{B} f(y) d y$ and $\Psi_{\theta^{\prime}}(B)=\left(1+r / \rho\left(x_{0}\right)\right)^{\theta^{\prime}}$, $B=B\left(x_{0}, r\right)$, and $\theta^{\prime}=\left(k_{0}+1\right) \theta$.

The dyadic maximal operator $M_{V}^{\Delta} f(x)$ is defined by

$$
M_{V}^{\Delta} f(x)=\sup _{x \in Q} \frac{1}{\Psi(Q)|Q|} \int_{Q}|f(x)| d x,
$$

where $Q$ is a dyadic cube.

The dyadic sharp maximal operator $M_{V}^{\sharp} f(x)$ is defined by

$$
\begin{aligned}
M_{V}^{\sharp} f(x)= & \sup _{x \in Q_{x_{0}}, r<\rho\left(x_{0}\right)} \frac{1}{\left|Q_{x_{0}}\right|} \int_{Q_{x_{0}}}\left|f(y)-f_{Q_{x_{0}}}\right| d y \\
& +\sup _{x \in Q_{x_{0}}, r \geq \rho\left(x_{0}\right)} \frac{1}{\Psi\left(Q_{x_{0}}\right)\left|Q_{x_{0}}\right|} \int_{Q_{x_{0}}}|f(y)| d y \\
\simeq & \sup _{x \in Q_{x_{0}}, r<\rho\left(x_{0}\right)} \inf _{C} \frac{1}{\left|Q_{x_{0}}\right|} \int_{Q_{x_{0}}}|f(y)-C| d y \\
& +\sup _{x \in Q_{x_{0}}, r \geq \rho\left(x_{0}\right)} \frac{1}{\Psi\left(Q_{x_{0}}\right)\left|Q_{x_{0}}\right|} \int_{Q_{x_{0}}}|f(y)| d y,
\end{aligned}
$$

where $Q_{x_{0}}$ denotes dyadic cubes $Q\left(x_{0}, r\right)$ and $f_{Q}=(1 /|Q|)$ $\int_{Q} f(x) d x$. 
A variant of dyadic maximal operator and dyadic sharp maximal operator is defined as following:

$$
\begin{aligned}
& M_{\delta, V}^{\Delta} f(x)=M_{V}^{\Delta}\left(|f|^{\delta}\right)^{1 / \delta}(x), \\
& M_{\delta, V}^{\sharp} f(x)=M_{V}^{\sharp}\left(|f|^{\delta}\right)^{1 / \delta}(x) .
\end{aligned}
$$
$\omega=1$.

In our paper, we need the following proposition when

Proposition 12 (see [15]). Let $1<p<\infty$ and suppose that $\omega \in A_{p}^{\rho}$. If $p<p_{1}<\infty$, then the equality

$$
\int_{R^{d}}\left|M_{V} f(x)\right|^{p_{1}} \omega(x) d x \leq C_{p} \int_{R^{d}}|f(x)|^{p_{1}} \omega(x) d x .
$$

Further, let $1 \leq p<\infty, \omega \in A_{p}^{\rho}$, if and only if

$$
\omega\left(\left\{x \in R^{d}: M_{V} f(x)>\lambda\right\}\right) \leq \frac{C_{p}}{\lambda^{p}} \int_{R^{d}}|f(x)|^{p} \omega(x) d x .
$$

A function $A:[0, \infty) \rightarrow[0, \infty)$ is said to be a Young function if it is continuous, convex, and increasing satisfying $A(0)=0$ and $A(t) \rightarrow \infty$ as $t \rightarrow \infty$. We defined the $A$-average of a function $f$ over a cube $Q$ by means of the following Luxemburg norm:

$$
\|f\|_{A, Q}=\inf \left\{\lambda>0: \frac{1}{|Q|} \int_{Q} A\left(\frac{|f(y)|}{\lambda}\right) d y \leq 1\right\} \text {, }
$$

and the maximal operator $M_{A}$ associated to $\|\cdot\|_{A, Q}$ by $M_{A} f(x)=\sup _{x \in \mathrm{Q}}\|f\|_{A, \mathrm{Q}}$. Then the following generalized Hölder inequality holds:

$$
\frac{1}{|Q|} \int_{Q}|f(y) g(y)| d y \leq\|f\|_{A, Q}\|g\|_{\bar{A}, Q^{\prime}}
$$

where $\bar{A}$ is the complementary Young function of $A$.

We define the corresponding maximal function

$$
\begin{gathered}
M_{A} f(x)=\sup _{x \in Q}\|f\|_{A, Q}, \\
M_{V, A} f(x)=\sup _{x \in Q} \Psi(Q)^{-1}\|f\|_{A, Q} .
\end{gathered}
$$

In [16], one has given a general result that can be applied to prove the boundedness of the localized classical operators. One considers a covering of balls $\left\{Q_{j}\right\}$ such that the family of a fixed dilation of them, $\left\{\widetilde{Q}_{j}\right\}$, has bounded overlapping (e.g. a covering associated to $\rho$ like in Proposition 3 ).

An operator $S$ is defined by

$$
S(f)=\sum_{j} \chi_{Q_{j}}\left|S\left(f \chi_{\widetilde{Q}_{j}}\right)\right| .
$$

Proposition 13 (see [16]). Let $1 \leq p \leq \nu<\infty$, and a weight $\omega$ on $R^{d}$ with the following property: for each $j,\left.\omega\right|_{\widetilde{Q}_{j}}$, admits an extension $\omega_{j}$ to $R^{d}$ such that

$$
S: L^{p}\left(\omega_{j}\right) \longmapsto L^{\nu}\left(\omega_{j}^{\nu / p}\right)
$$

bound with a constant independent of $j$. Then,

$$
S_{0}: L^{p}\left(\omega_{j}\right) \longmapsto L^{\nu}\left(\omega_{j}^{\nu / p}\right)
$$

continuously. If $p=1$, the assumption of $S$ is changed by weak type $(1, v)$, and the corresponding weak type can be concluded for $S_{0}$.

In our paper, set $S=\mu_{j}^{\Delta}$ and $\omega=1$. As we know, $\mu_{j}^{\Delta}$ is bounded from $L^{p}$ to $L^{p}$. So, Proposition 13 also holds for $\mu_{j}^{\Delta}$ and $\omega=1$.

From [16], we have the following result.

Lemma 14. Let $\left(\mu_{j}^{\Delta}\right)_{\mathrm{loc}}(f)(x)=\mu_{j}^{\Delta}\left(f \chi_{B(x, \rho(x))}\right)(x)$. Assume that $1<p<\infty$. Then there exists a constant $C>0$ such that

$$
\int_{R^{d}}\left|\left(\mu_{j}^{\Delta}\right)_{\mathrm{loc}}(f)(x)\right|^{p} d x \leq C \int_{R^{d}}|f(x)|^{p} d x .
$$

Furthermore, when $p=1$, there exists a constant $C$ such that, for all $\lambda>0$,

$$
\left|\left\{x \in R^{d}:\left(\mu_{j}^{\Delta}\right)_{\mathrm{loc}}(f)(x)>\lambda\right\}\right| \leq \frac{C}{\lambda} \int_{R^{d}}|f(x)| d x
$$

Proof. Let $\sigma=C 2^{k_{0} /\left(k_{0}+1\right)}$, with $C$ and $k_{0}$ as in Proposition 2 . Let $\left\{Q_{j}\right\}$ be the family given by Proposition 3 and set $\widetilde{Q}_{j}=$ $\sigma Q_{j}$.

Clearly, we have

$$
\bigcup_{x \in Q_{j}} B(x, \rho(x)) \subset \widetilde{Q}_{j}
$$

First, for $x \in Q_{j}$, Minkowski's inequality says that

$$
\begin{aligned}
& \left|\left(\mu_{j}^{\Delta}\right)_{\mathrm{loc}}(f)(x)-\mu_{j}^{\Delta}\left(\chi_{\widetilde{\mathrm{Q}}_{j}} f\right)(x)\right| \\
& \leq C\left(\int_{0}^{\infty} \mid \int_{|x-y| \leq t} K_{j}^{\Delta}(x, y) f(y)\right. \\
& \left.\quad \times\left.\left(\chi_{\widetilde{Q}_{j}}-\chi_{B(x, \rho(x))}\right)(y) d y\right|^{2} \frac{d t}{t^{3}}\right)^{1 / 2} \\
& \leq C \int_{R^{d}}\left|f(y)\left(\chi_{\widetilde{Q}_{j}}-\chi_{B(x, \rho(x))}\right)(y)\right| \\
& \quad \times\left|K_{j}^{\Delta}(x, y)\right|\left(\int_{|x-y| \leq t} \frac{d t}{t^{3}}\right)^{1 / 2} \\
& \leq C \int_{\widetilde{Q}_{j} \backslash B(x, \rho(x))}|f(y)| \frac{\left|x_{j}-y_{j}\right|}{|x-y|^{d}}|x-y|^{-1} d y
\end{aligned}
$$




$$
\begin{aligned}
& \leq C \int_{\widetilde{Q}_{j} \mid B(x, \rho(x))} \frac{|f(y)|}{|x-y|^{d}} d y \\
& \leq C \frac{1}{\left|\widetilde{Q}_{j}\right|} \int_{\widetilde{Q}_{j}}|f(y)| d y,
\end{aligned}
$$

where we have used $|x-y| \geq \rho(x) \simeq \rho\left(x_{j}\right)$ when $x \in Q_{j}$ and $y \notin B(x, \rho(x))$.

Therefore, for $1<p<\infty$,

$$
\begin{aligned}
& \left\|\left(\mu_{j}^{\Delta}\right)_{\mathrm{loc}}(f)\right\|_{L^{p}}^{p} \\
& \quad \leq C \sum_{j} \int_{\mathrm{Q}_{j}}\left(\frac{1}{\left|\widetilde{Q}_{j}\right|} \int_{\widetilde{Q}_{j}}|f(y)| d y\right)^{p} d x+\left\|\left(\mu_{j}^{\Delta}\right)_{0}(f)\right\|_{L^{p}}^{p} \\
& \quad \leq C \sum_{j} \int_{\mathrm{Q}_{j}} \frac{1}{\left|\widetilde{\mathrm{Q}}_{j}\right|} \int_{\widetilde{\mathrm{Q}}_{j}}|f(y)|^{p} d y d x+\left\|\left(\mu_{j}^{\Delta}\right)_{0}(f)\right\|_{L^{p}}^{p} \\
& \leq C \sum_{j} \int_{\widetilde{\mathrm{Q}}_{j}}|f(y)|^{p} d y+\left\|\left(\mu_{j}^{\Delta}\right)_{0}(f)\right\|_{L^{p}}^{p} \\
& \quad \leq C \int_{R^{d}}|f(y)|^{p} d y+\left\|\left(\mu_{j}^{\Delta}\right)_{0}(f)\right\|_{L^{p}}^{p} .
\end{aligned}
$$

Hence from Proposition 13 we have done it $1<p<\infty$.

For the case $p=1$, using the estimate of $\mid\left(\mu_{j}^{\Delta}\right)_{\text {loc }}(f)(x)-$ $\mu_{j}^{\Delta}\left(\chi_{\widetilde{Q}_{j}} f\right)(x) \mid$ again, we have, for each $j$,

$$
\begin{aligned}
& \left|\left\{x \in Q_{j}:\left|\left(\mu_{j}^{\Delta}\right)_{\text {loc }}(f)(x)-\mu_{j}^{\Delta}\left(\chi_{\widetilde{Q}_{j}} f\right)(x)\right|>\lambda\right\}\right| \\
& \leq C \frac{1}{\lambda} \int_{\widetilde{Q}_{j}}|f(y)| d y .
\end{aligned}
$$

Besides, from Proposition 13,

$$
\left|\left\{x \in Q_{j}:\left|\left(\mu_{j}^{\Delta}\right)_{0}\left(\chi_{\widetilde{Q}_{j}} f\right)(x)\right|>\lambda\right\}\right| \leq C \frac{1}{\lambda} \int_{\widetilde{Q}_{j}}|f(y)| d y .
$$

Therefore, summing over $j$ we have the weak type $(1,1)$.

Lemma 15. Let $b \in B M O_{\theta}(\rho)$, and $\left(k_{0}+1\right) \leq \eta<\infty$. Set $\left[b, \mu_{j}^{\Delta}\right]_{\mathrm{loc}}(f)(x)=\left[b, \mu_{j}^{\Delta}\right]\left(f \chi_{B(x, \rho(x))}\right)(x)$. Let $0<2 \delta<\epsilon<1$. Then,

$$
\begin{aligned}
& M_{\delta, \eta}^{\sharp}\left(\left[b, \mu_{j}^{\Delta}\right]_{\mathrm{loc}}(f)\right)(x) \\
& \leq C[b]_{\theta}\left(M_{\epsilon, \eta}^{\Delta}\left(\left(\mu_{j}^{\Delta}\right)_{\mathrm{loc}}(f)\right)(x)\right. \\
& \left.\quad+M_{L \log L, V, \eta}(f)(x)\right), \text { a.e. } x \in R^{d},
\end{aligned}
$$

holds for any $f \in C_{0}^{\infty}\left(R^{d}\right)$.
Proof. We fix $x \in R^{d}$ and let $x \in Q=Q\left(x_{0}, r\right)$ (dyadic cube). We consider two cases about $r$; that is, $r<\rho\left(x_{0}\right)$ and $r \geq$ $\rho\left(x_{0}\right)$.

Case 1. When $r<\rho\left(x_{0}\right)$, decomposing $f=f_{1}+f_{2}$, where $f_{1}=f \chi_{Q^{*}}$, where $Q^{*}=Q\left(x_{0}, 4 \sqrt{d} r\right)$. Let $C_{Q}$ a constant be fixed along the proof. Since $0<\delta<1$, we then have

$$
\begin{aligned}
& \left(\left.\frac{1}{|Q|} \int_{\mathrm{Q}}||\left[b, \mu_{j}^{\Delta}\right]_{\mathrm{loc}}(f)(y)\right|^{\delta}-\left|C_{\mathrm{Q}}\right|^{\delta} \mid d y\right)^{1 / \delta} \\
& \leq\left(\frac{1}{|\mathrm{Q}|} \int_{\mathrm{Q}}\left|\left[b, \mu_{j}^{\Delta}\right]_{\mathrm{loc}}(f)(y)-C_{\mathrm{Q}}\right|^{\delta} d y\right)^{1 / \delta} \\
& \leq C\left(\frac{1}{|\mathrm{Q}|} \int_{\mathrm{Q}}\left|\left(b(y)-b_{\mathrm{Q}^{*}}\right)\left(\mu_{j}^{\Delta}\right)_{\mathrm{loc}}(f)(y)\right|^{\delta} d y\right)^{1 / \delta} \\
& \quad+C\left(\frac{1}{|\mathrm{Q}|} \int_{\mathrm{Q}}\left|\left(\mu_{j}^{\Delta}\right)_{\mathrm{loc}}\left(\left(b-b_{\mathrm{Q}^{*}}\right) f_{1}\right)(y)\right|^{\delta} d y\right)^{1 / \delta} \\
& \quad+C\left(\frac{1}{|\mathrm{Q}|} \int_{\mathrm{Q}}\left|\left(\mu_{j}^{\Delta}\right)_{\mathrm{loc}}\left(\left(b-b_{\mathrm{Q}^{*}}\right) f_{2}\right)(y)-C_{\mathrm{Q}}\right|^{\delta} d y\right)^{1 / \delta} \\
& =\mathrm{I}+\mathrm{II}+\mathrm{III},
\end{aligned}
$$

where $b_{\mathrm{Q}^{*}}=\left(1 /\left|\mathrm{Q}^{*}\right|\right) \int_{\mathrm{Q}^{*}} b(y) d y$.

We start with I. For any $1<\gamma<\epsilon / \delta$, note that $M_{V}(x) \sim$ $M_{V}\left(x_{0}\right)$ for any $x \in Q^{*}$ and $\Psi\left(Q^{*}\right) \sim 1$; by Lemma 9 , we obtain

$$
\begin{aligned}
\mathrm{I} \leq & \left(\frac{1}{\left|Q^{*}\right|} \int_{\mathrm{Q}^{*}}\left|b(y)-b_{\mathrm{Q}^{*}}\right|^{\delta \gamma^{\prime}} d y\right)^{1 /\left(\delta \gamma^{\prime}\right)} \\
& \times\left(\frac{1}{|\mathrm{Q}|} \int_{\mathrm{Q}}\left|\left(\mu_{j}^{\Delta}\right)_{\mathrm{loc}}(f)(y)\right|^{\delta \gamma} d y\right)^{1 /(\delta \gamma)} \\
& \leq C[b]_{\theta} M_{\epsilon, \eta}^{\Delta}\left(\left(\mu_{j}^{\Delta}\right)_{\mathrm{loc}}(f)\right)(x),
\end{aligned}
$$

where $1 / \gamma+1 / \gamma^{\prime}=1$

For II, note that $M_{V}(x) \sim M_{V}\left(x_{0}\right)$ for any $x \in Q^{*}$ and $\Psi\left(Q^{*}\right) \sim 1$; by Kolmogorov's inequality, Proposition 11, and Lemma 14, we have

$$
\begin{aligned}
\mathrm{II} & \leq \frac{C}{|Q|}\left\|\left(\mu_{j}^{\Delta}\right)_{\mathrm{loc}}\left(\left(b-b_{\mathrm{Q}^{*}}\right) f_{1}\right)\right\|_{L^{1, \infty}} \\
& \leq \frac{C}{\left|Q^{*}\right|} \int_{\mathrm{Q}^{*}}\left|\left(b(y)-b_{\mathrm{Q}^{*}}\right) f(y)\right| d y \\
& \leq C[b]_{\theta} M_{L \log L, V, \eta}(f)(x) .
\end{aligned}
$$

To deal with III, we first fix the value of $C_{\mathrm{Q}}$ by taking $C_{\mathrm{Q}}=$ $\left(\mu_{j}^{\Delta}\right)_{\text {loc }}\left(\left(b-b_{Q^{*}}\right) f_{2}\right)\left(y_{0}\right)$ with $y_{0} \in Q$; we have

$$
\begin{gathered}
\left|\left(\mu_{j}^{\Delta}\right)_{\mathrm{loc}}\left(\left(b-b_{\mathrm{Q}^{*}}\right) f_{2}\right)(y)-\left(\mu_{j}^{\Delta}\right)_{\mathrm{loc}}\left(\left(b-b_{\mathrm{Q}^{*}}\right) f_{2}\right)\left(y_{0}\right)\right| \\
\leq\left(\int_{0}^{\infty}\left|\int_{|z-y| \leq t<\left|z-y_{0}\right|}\right| K_{j}^{\Delta}(y, z)\left(b(z)-b_{\mathrm{Q}^{*}}\right)\right. \\
\left.\times f_{2}(z) \chi_{B(y, \rho(y))}|d z|^{2} \frac{d t}{t^{3}}\right)^{1 / 2}
\end{gathered}
$$




$$
\begin{aligned}
& +\left(\int_{0}^{\infty}\left|\int_{\left|z-y_{0}\right| \leq t<|z-y|}\right| K_{j}^{\Delta}\left(y_{0}, z\right)\left(b(z)-b_{Q^{*}}\right)\right. \\
& \left.\times f_{2}(z) \chi_{B\left(y_{0}, \rho\left(y_{0}\right)\right)}|d z|^{2} \frac{d t}{t^{3}}\right)^{1 / 2} \\
& +\left(\int_{0}^{\infty}\left|\int_{\left\{\left|z-y_{0}\right| \leq t,|z-y| \leq t\right\}}\right| K_{j}^{\Delta}(y, z)-K_{j}^{\Delta}\left(y_{0}, z\right) \mid\right. \\
& \times \mid\left(b(z)-b_{Q^{*}}\right) f_{2}(z) \\
& \left.\times \chi_{B\left(y_{0}, \rho\left(y_{0}\right)\right)}|d z|^{2} \frac{d t}{t^{3}}\right)^{1 / 2} \\
& +\left(\int_{0}^{\infty}\left|\int_{\left\{\left|z-y_{0}\right| \leq t,|z-y| \leq t\right\}}\right| K_{j}^{\Delta}(y, z)\left(b(z)-b_{Q^{*}}\right)\right. \\
& \left.\times f_{2}(z) \chi_{B\left(y_{0}, \rho\left(y_{0}\right)\right)}|d z|^{2} \frac{d t}{t^{3}}\right)^{1 / 2} \\
& +\left(\int_{0}^{\infty}\left|\int_{\left\{\left|z-y_{0}\right| \leq t,|z-y| \leq t\right\}}\right| K_{j}^{\Delta}(y, z)\left(b(z)-b_{\mathrm{Q}^{*}}\right)\right. \\
& \left.\times f_{2}(z) \chi_{B(y, \rho(y))}|d z|^{2} \frac{d t}{t^{3}}\right)^{1 / 2} \\
& =E_{1}+E_{2}+E_{3}+E_{4}+E_{5} .
\end{aligned}
$$

For $E_{1}$, since $\rho(y) \sim \rho\left(x_{0}\right)$ and $|z-y| \sim\left|z-y_{0}\right| \sim\left|z-x_{0}\right|$, using Minkowski's inequality and Proposition 11, we obtain

$$
\begin{aligned}
& E_{1} \leq C \int_{R^{d}}\left|K_{j}^{\Delta}(y, z)\left(b(z)-b_{Q^{*}}\right) f_{2}(z) \chi_{B(y, \rho(y))}\right| \\
& \times\left(\int_{|z-y| \leq t<\left|z-y_{0}\right|} \frac{d t}{t^{3}}\right)^{1 / 2} d z \\
& \leq C r^{1 / 2} \int_{\left(\mathrm{Q}^{*}\right)^{c} \cap B(y, \rho(y))}\left|\left(b(z)-b_{\mathrm{Q}^{*}}\right) f(z)\right| \\
& \times \frac{\left|z_{j}-y_{j}\right| /|z-y|}{|z-y|^{d-1}} \frac{1}{|z-y|^{3 / 2}} d z \\
& \leq C r^{1 / 2} \int_{\left(Q^{*}\right)^{c} \cap B(y, \rho(y))}\left|\left(b(z)-b_{Q^{*}}\right) f(z)\right| \\
& \times \frac{1}{|z-y|^{d+1 / 2}} d z \\
& \leq C r^{1 / 2} \int_{4 \sqrt{d} r<\left|z-x_{0}\right| \leq \rho\left(x_{0}\right)}\left|\left(b(z)-b_{Q^{*}}\right) f(z)\right| \\
& \times \frac{1}{\left|z-x_{0}\right|^{d+1 / 2}} d z \\
& \leq C \sum_{k=2}^{N_{0}} \frac{2^{-k / 2}}{\left(2^{k} \sqrt{d} r\right)^{d}} \int_{\left|z-x_{0}\right| \leq 2^{k+1} \sqrt{d} r}\left|\left(b(z)-b_{\mathrm{Q}^{*}}\right) f(z)\right| d z \\
& \leq C[b]_{\theta} M_{L \log L, V, \eta}(f)(x) \text {, }
\end{aligned}
$$

where the integer $k_{0}$ satisfies $2^{N_{0}} \sqrt{d} r \leq \rho\left(x_{0}\right) \leq 2^{N_{0}+1} \sqrt{d} r$.
Similarly,

$$
E_{2} \leq C[b]_{\theta} M_{L \log L, V, \eta}(f)(x) .
$$

For $E_{3}$, since $\rho\left(y_{0}\right) \sim \rho\left(x_{0}\right)$ and $|z-y| \sim\left|z-y_{0}\right| \sim\left|z-x_{0}\right|$, by Minkowski's inequality and Proposition 11, we have

$$
\begin{aligned}
E_{3} \leq & C \int_{R^{d}}\left|K_{j}^{\Delta}(y, z)-K_{j}^{\Delta}\left(y_{0}, z\right)\right| \\
& \left.\times \mid\left(b(z)-b_{\mathrm{Q}^{*}}\right) f_{2}(z) \chi_{B\left(y_{0}, \rho\left(y_{0}\right)\right) \mid} \frac{d t}{t^{3}}\right)^{1 / 2} d z \\
& \times\left(\int_{\left\{\left|z-y_{0}\right| \leq t,|z-y| \leq t\right\}}\left|\left(b(z)-b_{\mathrm{Q}^{*}}\right) f(z)\right|\right. \\
\leq & C \int_{\left(\mathrm{Q}^{*}\right)^{c} \cap B\left(y_{0}, \rho\left(y_{0}\right)\right)} \\
& \times \frac{\left|y_{j}-y_{0 j}\right|}{\left|z-y_{0}\right|^{d}} \frac{1}{\left|z-y_{0}\right|} d z \\
\leq & C \int_{4 \sqrt{d} r<\left|z-x_{0}\right| \leq \rho\left(x_{0}\right)}\left|\left(b(z)-b_{\mathrm{Q}^{*}}\right) f(z)\right| \frac{\left|y-y_{0}\right|}{\left|z-x_{0}\right|^{d+1}} d z \\
\leq & C \sum_{k=2}^{N_{0}} \frac{r\left(2^{k} \sqrt{d} r\right)^{d+1}}{\leq} \int_{\left|z-x_{0}\right| \leq 2^{k+1} \sqrt{d} r}\left|\left(b(z)-b_{\mathrm{Q}^{*}}\right) f(z)\right| d z \\
\leq & C \sum_{k=2}^{N_{0}} \frac{2^{-k}}{\left(2^{k} \sqrt{d} r\right)_{\theta}^{d} M_{L \log L, V, \eta}(f)(x),}\left|\left(b(z)-b_{\mathrm{Q}^{*}}\right) f(z)\right| d z \\
&
\end{aligned}
$$

where the integer $N_{0}$ is the same as above.

For $E_{4}$, since $\rho\left(y_{0}\right) \sim \rho\left(x_{0}\right)$ and $|z-y| \sim\left|z-y_{0}\right| \sim\left|z-x_{0}\right|$, using Minkowski's inequality and Proposition 11, we obtain

$$
\begin{aligned}
E_{4} \leq & C \int_{R^{d}}\left|K_{j}^{\Delta}(y, z)\left(b(z)-b_{\mathrm{Q}^{*}}\right) f_{2}(z) \chi_{B\left(y_{0}, \rho\left(y_{0}\right)\right)}\right| \\
& \times\left(\int_{\left\{\left|z-y_{0}\right| \leq t,|z-y| \leq t\right\}} \frac{d t}{t^{3}}\right)^{1 / 2} d z \\
\leq & C \int_{\left(Q^{*}\right)^{c} \cap B\left(y_{0}, \rho\left(y_{0}\right)\right)}\left|\left(b(z)-b_{\mathrm{Q}^{*}}\right) f(z)\right| \\
\leq & \times \frac{\left|z_{j}-y_{j}\right| /|z-y|}{|z-y|^{d-1}} \frac{1}{|z-y|} d z \\
\leq & \int_{4 \sqrt{d} r<\left|z-x_{0}\right| \leq \rho\left(x_{0}\right)}\left|\left(b(z)-b_{\mathrm{Q}^{*}}\right) f(z)\right| \frac{1}{\left|z-x_{0}\right|^{d}} d z \\
\leq & C \sum_{k=2}^{N_{0}} \frac{1}{\left(2^{k} \sqrt{d} r\right)^{d}} \int_{\left|z-x_{0}\right| \leq 2^{k+1} \sqrt{d} r}\left|\left(b(z)-b_{\mathrm{Q}^{*}}\right) f(z)\right| d z \\
\leq & C[b]_{\theta} M_{L \log L, V, \eta}(f)(x),
\end{aligned}
$$

where the integer $N_{0}$ is the same as above, and we know it is finite. 
Similarly,

$$
E_{5} \leq C[b]_{\theta} M_{L \log L, V, \eta}(f)(x) .
$$

So,

$$
\begin{aligned}
\mathrm{III} & \leq \frac{C}{|\mathrm{Q}|} \int_{\mathrm{Q}} \mid\left(\mu_{j}^{\Delta}\right)_{\mathrm{loc}}\left(\left(b-b_{\mathrm{Q}^{*}}\right) f_{2}\right)(y) \\
& -\left(\mu_{j}^{\Delta}\right)_{\mathrm{loc}}\left(\left(b-b_{\mathrm{Q}^{*}}\right) f_{2}\right)\left(y_{0}\right) \mid d y \\
& \leq C[b]_{\theta} M_{L \log L, V, \eta}(f)(x) .
\end{aligned}
$$

Case 2. When $r \geq \rho\left(x_{0}\right)$, decomposing $f=f_{1}+f_{2}$, where $f_{1}=f \chi_{Q^{*}}$, where $Q^{*}=Q\left(x_{0}, C_{0} 2^{k_{0}+4} \sqrt{d} r\right)$. Since $0<2 \delta<$ $\epsilon<1$, so $a=\eta / \delta$ and $\epsilon / \delta>2$; then,

$$
\begin{aligned}
& \frac{1}{\Psi(Q)^{a}}\left(\frac{1}{|\mathrm{Q}|} \int_{\mathrm{Q}}\left|\left[b, \mu_{j}^{\Delta}\right]_{\mathrm{loc}}(f)(y)\right|^{\delta} d y\right)^{1 / \delta} \\
& \leq \frac{1}{\Psi(Q)^{a}}\left(\frac{1}{|\mathrm{Q}|} \int_{\mathrm{Q}} \mid\left(b(y)-b_{\mathrm{Q}^{*}}\right)\left(\mu_{j}^{\Delta}\right)_{\mathrm{loc}}(f)(y)\right. \\
& \left.+\left.\left(\mu_{j}^{\Delta}\right)_{\mathrm{loc}}\left(\left(b-b_{\mathrm{Q}^{*}}\right) f\right)(y)\right|^{\delta} d y\right)^{1 / \delta} \\
& \leq C \frac{1}{\Psi(\mathrm{Q})^{a}}\left(\frac{1}{|\mathrm{Q}|} \int_{\mathrm{Q}}\left|\left(b(y)-b_{\mathrm{Q}^{*}}\right)\left(\mu_{j}^{\Delta}\right)_{\mathrm{loc}}(f)(y)\right|^{\delta} d y\right)^{1 / \delta} \\
& +C \frac{1}{\Psi(\mathrm{Q})^{a}}\left(\frac{1}{|\mathrm{Q}|} \int_{\mathrm{Q}}\left|\left(\mu_{j}^{\Delta}\right)_{\mathrm{loc}}\left(\left(b-b_{\mathrm{Q}^{*}}\right) f_{1}\right)(y)\right|^{\delta} d y\right)^{1 / \delta} \\
& +C \frac{1}{\Psi(\mathrm{Q})^{a}}\left(\frac{1}{|\mathrm{Q}|} \int_{\mathrm{Q}}\left|\left(\mu_{j}^{\Delta}\right)_{\mathrm{loc}}\left(\left(b-b_{\mathrm{Q}^{*}}\right) f_{2}\right)(y)\right|^{\delta} d y\right)^{1 / \delta} \\
& =\mathrm{I}+\mathrm{II}+\mathrm{III},
\end{aligned}
$$

where $b_{\mathrm{Q}^{*}}=\left(1 /\left|\mathrm{Q}^{*}\right|\right) \int_{\mathrm{Q}^{*}} b(y) d y$.

To deal with I, for any $2 \leq \gamma<\epsilon / \delta$, note that $k_{0}+1 \leq \eta$; by Lemma 9 , we then have

$$
\begin{aligned}
\mathrm{I} \leq & C \frac{1}{\Psi_{\theta^{\prime}}(Q)}\left(\frac{1}{\left|Q^{*}\right|} \int_{Q^{*}}\left|b(y)-b_{Q^{*}}\right|^{\delta \gamma^{\prime}} d y\right)^{1 /\left(\delta \gamma^{\prime}\right)} \\
& \times \frac{\Psi_{\theta^{\prime}}(Q)}{\Psi(Q)^{a-\eta /(2 \delta)}} \\
& \times\left(\frac{1}{\Psi(Q)^{\eta}|Q|} \int_{Q}\left|\left(\mu_{j}^{\Delta}\right)_{\mathrm{loc}}(f)(y)\right|^{\delta \gamma} d y\right)^{1 /(\delta \gamma)} \\
\leq & C[b]_{\theta} M_{\epsilon, \eta}^{\Delta}\left(\left(\mu_{j}^{\Delta}\right)_{\mathrm{loc}}(f)\right)(x),
\end{aligned}
$$

where $1 / \gamma+1 / \gamma^{\prime}=1$.
For II, using Kolmogorov's inequality, Proposition 11, and Lemma 14, we have

$$
\begin{aligned}
\mathrm{II} & \leq \frac{C}{\Psi(Q)^{a}|Q|}\left\|\left(\mu_{j}^{\Delta}\right)_{\mathrm{loc}}\left(\left(b-b_{\mathrm{Q}^{*}}\right) f_{1}\right)\right\|_{L^{1, \infty}} \\
& \leq \frac{C}{\Psi(Q)^{a}\left|Q^{*}\right|} \int_{Q^{*}}\left|\left(b(y)-b_{\mathrm{Q}^{*}}\right) f(y)\right| d y \\
& \leq C[b]_{\theta} M_{L \log L, V, \eta}(f)(x) .
\end{aligned}
$$

Finally, for III, notice that $B(y, \rho(y)) \subset Q\left(x_{0}, C_{0} 2^{k_{0}+4} \sqrt{d} r\right)$ for any $y \in Q$; then III $=0$.

Hence the proof is finished.

The following information can be founded in [4]. Define the following maximal functions:

$$
\begin{aligned}
& M_{V, \eta} f(x)= \sup _{x \in B} \frac{1}{(\Psi(B))^{\eta}|B|} \int_{B}|f(y)| d y, \\
& \widetilde{M}_{V, \eta} f(x)=\sup _{\epsilon>0} \frac{1}{(1+\epsilon \psi(B(x, \epsilon)))^{\theta \eta}} \\
& \times \int_{R^{d}} \epsilon^{-n} \varphi\left(\frac{x-y}{\epsilon}\right)|f(y)| d y,
\end{aligned}
$$

and their commutators

$$
\begin{aligned}
& M_{V, \eta}^{b} f(x)=\sup _{x \in B} \frac{1}{(\Psi(B))^{\eta}|B|} \int_{B}|b(x)-b(y)||f(y)| d y, \\
& \widetilde{M}_{V, \eta}^{b} f(x) \\
&=\sup _{\epsilon>0} \frac{1}{(1+\epsilon \psi(B(x, \epsilon)))^{\theta \eta}} \\
& \quad \times \int_{R^{d}} \epsilon^{-n} \varphi\left(\frac{x-y}{\epsilon}\right)|b(x)-b(y)||f(y)| d y,
\end{aligned}
$$

where $\psi(B(x, \epsilon))=(1 /|B(x, \epsilon)|) \int_{B(x, \epsilon)} \rho(y)^{-1} d y$.

Obviously, we have

$$
M_{V, \eta^{\prime}}^{b} f(x) \leq C \widetilde{M}_{V, \eta}^{b} f(x),
$$

where $\eta^{\prime}=\left(k_{0}+1\right) \eta$ and $\eta>0$.

Lemma 16 (see [1]). Let $b \in B M O_{\theta}(\rho)$, and $\left(k_{0}+1\right)(1+1 / \theta) \leq$ $\eta<\infty, \eta_{1}=\left(k_{0}+1\right) \eta$, and $\eta_{2}=\left(k_{0}+1\right) \eta_{1}(1+1 / \theta)$. Let $0<2 \delta<\epsilon<1$; then,

$$
\begin{aligned}
M_{\delta, \eta}^{\sharp}\left(\widetilde{M}_{V, \eta_{2}}^{b}(f)\right)(x) & \leq C[b]_{\theta}\left(M_{\epsilon, \eta}^{\Delta}\left(\widetilde{M}_{V, \eta_{2}}(f)\right)(x)\right. \\
& \left.+M_{L \log L, V, \eta}(f)(x)\right), \quad \text { a.e } x \in R^{d},
\end{aligned}
$$

holds for any $f \in C_{0}^{\infty}\left(R^{d}\right)$.

In the following Lemma, we set $\omega=1$. 
Lemma 17 (see [4]). Let $2 \leq \eta<\infty, \omega \in A_{1}^{\rho}$, and $A(t)=$ $t \log (e+t)$. Then there exists a constant $C>0$ such that for all $t>0$

$$
\begin{aligned}
& \omega\left(\left\{x \in R^{d}: M_{A, V, \eta} f(x)>t\right\}\right) \\
& \quad \leq C \int_{R^{d}} A\left(\frac{|f(x)|}{t}\right) \omega(x) d x .
\end{aligned}
$$

Lemma 18 (see [15]). Let $0<\eta<\infty$ and let $M_{V, \eta / 2} f$ be locally integral. Then there exist positive constants $C_{1}$ and $C_{2}$ independent of $f$ and $x$ such that

$$
\begin{aligned}
C_{1} M_{V, \eta} M_{V, \eta+1} f(x) & \leq M_{L \log L, V, \eta+1} f(x) \\
& \leq C_{2} M_{V, \eta / 2} M_{V, \eta / 2} f(x) .
\end{aligned}
$$

\section{Proof of the Main Theorems}

Proof of Theorem 6. (i) Without loss of generality, we may assume that $\alpha<0$. Pick any $x_{0} \in R^{d}$ and $r>0$, and write

$$
f(x)=f_{0}(x)+\sum_{i=1}^{\infty} f_{i}(x)
$$

where $f_{0}=\chi_{B\left(x_{0}, 2 r\right)}$ and $f_{i}=\chi_{B\left(x_{0}, 2^{i+1} r\right) \backslash B\left(x_{0}, 2^{i} r\right)}$, for $i \geq 1$. Hence, we have

$$
\begin{aligned}
& \left(\int_{B\left(x_{0}, r\right)}\left|\mu_{j}^{L} f(x)\right|^{p} d x\right)^{1 / p} \\
& \leq C\left(\int_{B\left(x_{0}, r\right)}\left|\mu_{j}^{L} f_{0}(x)\right|^{p} d x\right)^{1 / p} \\
& \quad+\sum_{i=1}^{\infty}\left(\int_{B\left(x_{0}, r\right)}\left|\mu_{j}^{L} f_{i}(x)\right|^{p} d x\right)^{1 / p} .
\end{aligned}
$$

By the $L^{p}$ boundedness of $\mu_{j}^{L}$, we obtain

$$
\begin{aligned}
& \left(\int_{B\left(x_{0}, r\right)}\left|\mu_{j}^{L} f_{0}(x)\right|^{p} d x\right)^{1 / p} \\
& \quad \leq C\left(1+\frac{r}{\rho\left(x_{0}\right)}\right)^{-\alpha}|B|^{1 / p-1 / q}\|f\|_{M_{p, V}^{q, \alpha}\left(R^{d}\right)} .
\end{aligned}
$$

By Proposition 2, Lemma 8, and Minkowski's inequality, we have

$$
\begin{gathered}
\left(\int_{B\left(x_{0}, r\right)}\left|\mu_{j}^{L} f_{i}(x)\right|^{p} d x\right)^{1 / p} \\
\leq C\left(\int _ { B ( x _ { 0 } , r ) } \left(\int_{0}^{\infty} \mid \int_{|x-y| \leq t} K_{j}^{L}(x, y)\right.\right. \\
\leq C_{k}\left(\int _ { B ( x _ { 0 } , r ) } \left(\int_{R^{d}} \frac{\left.\left.\left|f_{i}(y) d y\right|^{2} \frac{d t}{t^{3}}\right)^{p / 2} d x\right)^{1 / p}}{(1+|x-y| / \rho(x))^{k}} \frac{1}{|x-y|^{d-1}}\right.\right. \\
\left.\left.\times\left(\int_{|x-y| \leq t} \frac{d t}{t^{3}}\right)^{1 / 2} d y\right)^{p} d x\right)^{1 / p}
\end{gathered}
$$

$\leq C_{k}\left(\int_{B\left(x_{0}, r\right)}\left(\int_{B\left(x_{0}, 2^{i+1} r\right) \backslash B\left(x_{0}, 2^{i} r\right)} \frac{|f(y)|}{(1+|x-y| / \rho(x))^{k}}\right.\right.$

$$
\left.\left.\times \frac{1}{|x-y|^{d}} d y\right)^{p} d x\right)^{1 / p}
$$

$$
\leq C_{k}\left(\int_{B\left(x_{0}, r\right)} \frac{\left(2^{i} r\right)^{-d}}{\left(1+2^{i} r / \rho(x)\right)^{k p}} d x\right.
$$$$
\left.\times \int_{B\left(x_{0}, 2^{i+1} r\right)}|f(y)|^{p} d y\right)^{1 / p}
$$$$
\begin{aligned}
& \leq C_{k}\left(\int_{B\left(x_{0}, r\right)} \frac{\left(2^{i} r\right)^{-d}}{\left(1+2^{i} r / \rho(x)\right)^{k p}} d x\right)^{1 / p} \\
& \quad \times\left(1+\frac{2^{i} r}{\rho\left(x_{0}\right)}\right)^{-\alpha}\left|2^{i} B\right|^{1 / p-1 / q}\|f\|_{M_{p, V}^{q, \alpha}\left(R^{d}\right)}
\end{aligned}
$$$$
\leq C_{k}\left(\int _ { B ( x _ { 0 } , r ) } \left(\left(2^{i} r\right)^{-d}\right.\right.
$$

$$
\begin{gathered}
\times\left(1+\frac{2^{i} r}{\rho\left(x_{0}\right)}\right. \\
\left.\left.\left.\times\left(1+\frac{r}{\rho\left(x_{0}\right)}\right)^{-k_{0} /\left(k_{0}+1\right)}\right)^{-k p}\right) d x\right)^{1 / p} \\
\times\left(1+\frac{2^{i} r}{\rho\left(x_{0}\right)}\right)^{-\alpha}\left|2^{i} B\right|^{1 / p-1 / q}\|f\|_{M_{p, V}^{q, \alpha}\left(R^{d}\right)} \\
\leq C_{k}\left(2^{-i}\right)^{d / p} \frac{\left(1+2^{i} r / \rho\left(x_{0}\right)\right)^{-\alpha}}{\left(1+2^{i} r / \rho\left(x_{0}\right)\right)^{k /\left(k_{0}+1\right)}}\left|2^{i} B\right|^{1 / p-1 / q} \\
\times\|f\|_{M_{p, V}^{q, \alpha}\left(R^{d}\right)} .
\end{gathered}
$$

Let $k=(-[\alpha]+1)\left(k_{0}+1\right)$, and we obtain

$$
\left\|\mu_{j}^{L} f\right\|_{M_{p, V}^{q, \alpha}\left(R^{d}\right)} \leq C\|f\|_{M_{p, V}^{q, \alpha}\left(R^{d}\right)} .
$$

(ii) When $p=1$ and noting that $\mu_{j}^{L}$ are bounded from $L^{1}\left(R^{d}\right)$ to weak $L^{1}\left(R^{d}\right)$, we have

$$
\begin{aligned}
\lambda\left|\left\{y \in B(x, r):\left|\mu_{j}^{L} f(y)\right|>\lambda\right\}\right| \\
\quad \leq \int_{B(x, r)}|f(y)| d y \\
\quad \leq C\left(1+\frac{r}{\rho\left(x_{0}\right)}\right)^{-\alpha}|B(x, r)|^{1-1 / q}\|f\|_{M_{1, V}^{q, \alpha}\left(R^{d}\right)^{.}}
\end{aligned}
$$

We have finished the proof of Theorem 6 . 
Journal of Function Spaces

9

Proof of Theorem 7. (i) Pick any $x_{0} \in R^{d}$ and $r>0$, as in Theorem 6; we write

$$
f(x)=f_{0}(x)+\sum_{i=1}^{\infty} f_{i}(x)
$$

and, by the $L^{p}$ boundedness of $\left[b, \mu_{j}^{L}\right]$, we obtain

$$
\begin{aligned}
& \left(\int_{B\left(x_{0}, r\right)}\left|\left[b, \mu_{j}^{L}\right] f_{0}(x)\right|^{p} d x\right)^{1 / p} \\
& \quad \leq C\left(1+\frac{r}{\rho\left(x_{0}\right)}\right)^{-\alpha}|B|^{1 / p-1 / q}\|f\|_{M_{p, V}^{q, \alpha}\left(R^{d}\right)}
\end{aligned}
$$

Set $b_{r}=\left(1 /\left|B\left(x_{0}, r\right)\right|\right) \int_{B\left(x_{0}, r\right)} b(x) d x$.

We write

$$
\left|\left[b, \mu_{j}^{L}\right] f_{i}\right| \leq\left|b-b_{r}\right|\left|\mu_{j}^{L} f_{i}\right|+\left|\mu_{j}^{L}\left(\left(b-b_{r}\right) f_{i}\right)\right| .
$$

When $i \geq 1$, by Lemmas 8 and 9, Proposition 2, Corollary 10, and Minkowski's inequality, we have

$$
\begin{aligned}
\left(\int_{B\left(x_{0}, r\right)}\left|\left[b, \mu_{j}^{L}\right] f_{i}(x)\right|^{p} d x\right)^{1 / p} & \\
\leq & \left(\int_{B\left(x_{0}, r\right)}\left|b(x)-b_{r}\right|^{p}\left|\mu_{j}^{L} f_{i}(x)\right|^{p} d x\right)^{1 / p} \\
& +\left(\int_{B\left(x_{0}, r\right)}\left|\mu_{j}^{L}\left(\left(b-b_{r}\right) f_{i}\right)(x)\right|^{p} d x\right)^{1 / p} \\
\leq & \left(\int_{B\left(x_{0}, r\right)}\left|b(x)-b_{r}\right|^{p}\right. \\
& \left.\times\left(\int_{0}^{\infty}\left|\int_{|x-y| \leq t} K_{j}^{L}(x, y) f_{i}(y) d y\right|^{2} \frac{d t}{t^{3}}\right)^{p / 2} d x\right)^{1 / p} \\
& +\left(\int _ { B ( x _ { 0 } , r ) } \left(\int_{0}^{\infty} \mid \int_{|x-y| \leq t} K_{j}^{L}(x, y)\left(b(y)-b_{r}\right)\right.\right. \\
\leq & \left.\left.\frac{C_{k}\left(2^{i} r\right)^{-d}}{\left(1+2^{i} r / \rho\left(x_{0}\right)\right)^{k /\left(k_{0}+1\right)}} \times\left. f_{B}(x) d y\right|^{2} \frac{d t}{t^{3}}\right)^{p / 2} d x\right)^{1 / p}
\end{aligned}
$$

$$
\begin{aligned}
& \leq \frac{C_{k}\left(2^{i} r\right)^{-d}}{\left(1+2^{i} r / \rho\left(x_{0}\right)\right)^{k /\left(k_{0}+1\right)}} \\
& \quad \times\left\{\left(\int_{B\left(x_{0}, r\right)}\left|b(x)-b_{r}\right|^{p} d x\right)^{1 / p}\right.
\end{aligned}
$$$$
\times\left(\int_{B\left(x_{0}, 2^{i+1} r\right)}|f(y)|^{p} d y\right)^{1 / p}\left(2^{i} r\right)^{d(1-1 / p)}
$$$$
+r^{d / p}\left(\int_{B\left(x_{0}, 2^{i+1} r\right)}\left|b(y)-b_{r}\right|^{p^{\prime}} d y\right)^{1 / p^{\prime}}
$$$$
\left.\times\left(\int_{B\left(x_{0}, 2^{i+1} r\right)}|f(y)|^{p} d y\right)^{1 / p}\right\}
$$$$
\leq \frac{C_{k}\left(2^{i} r\right)^{-d}}{\left(1+2^{i} r / \rho\left(x_{0}\right)\right)^{k /\left(k_{0}+1\right)}}
$$$$
\times\left\{[b]_{\theta} r^{d / p}\left(1+\frac{r}{\rho\left(x_{0}\right)}\right)^{\theta^{\prime}}\left(2^{i} r\right)^{d(1-1 / p)}\right.
$$$$
\times\left(1+\frac{2^{i} r}{\rho\left(x_{0}\right)}\right)^{-\alpha}\left|2^{i} B\right|^{1 / p-1 / q}\|f\|_{M_{p, V}^{q, \alpha}\left(R^{d}\right)}
$$$$
+r^{d / p} i[b]_{\theta}\left(1+\frac{r}{\rho\left(x_{0}\right)}\right)^{\theta^{\prime}}\left(2^{i} r\right)^{d(1-1 / p)}
$$$$
\left.\times\left(1+\frac{2^{i} r}{\rho\left(x_{0}\right)}\right)^{-\alpha}\left|2^{i} B\right|^{1 / p-1 / q}\|f\|_{M_{p, V}^{q, \alpha}\left(R^{d}\right)}\right\}
$$

$$
\begin{aligned}
\leq & C_{k} i[b]_{\theta}\left(2^{i}\right)^{-d / p} \frac{\left(1+2^{i} r / \rho\left(x_{0}\right)\right)^{-\alpha}}{\left(1+2^{i} r / \rho\left(x_{0}\right)\right)^{k /\left(k_{0}+1\right)-\theta^{\prime}}} \\
& \times\left|2^{i} B\right|^{1 / p-1 / q}\|f\|_{M_{p, V}^{q, \alpha}\left(R^{d}\right)},
\end{aligned}
$$

where $1 / p+1 / p^{\prime}=1$. Choosing $k$ large enough such that $k /\left(k_{0}+1\right)-\theta^{\prime}>0$, we obtain

$$
\left\|\left[b, \mu_{j}^{L}\right] f\right\|_{M_{p, V}^{q, \alpha}\left(R^{d}\right)} \leq C[b]_{\theta}\|f\|_{M_{p, V}^{q, \alpha}\left(R^{d}\right)} .
$$

(ii) We adapt a similar argument to that of Theorem 1.2 in [5].

Define

$$
\begin{gathered}
{\left[b, \mu_{j}^{L}\right]_{\mathrm{loc}}(f)(x)=\left[b, \mu_{j}^{L}\right]\left(f \chi_{B(x, \rho(x))}\right)(x),} \\
{\left[b, \mu_{j}^{L}\right]_{\mathrm{glob}}(f)(x)=\left[b, \mu_{j}^{L}\right]\left(f \chi_{B^{c}(x, \rho(x))}\right)(x) .}
\end{gathered}
$$



have

We start with $\left[b, \mu_{j}^{L}\right]_{\text {glob }}$. Using Lemma 8 with $N=1$, we

$$
\begin{aligned}
& {\left[b, \mu_{j}^{L}\right]_{\mathrm{glob}}(f)(x)} \\
& =\left(\int_{0}^{\infty} \mid \int_{|x-y| \leq t} K_{j}^{L}(x, y)(b(x)-b(y))\right. \\
& \left.\times\left. f(y) \chi_{B^{c}(x, \rho(x))} d y\right|^{2} \frac{d t}{t^{3}}\right)^{1 / 2} \\
& \leq\left(\int_{\rho(x)}^{\infty} \mid \int_{\rho(x)<|x-y| \leq t} K_{j}^{L}(x, y)(b(x)-b(y))\right. \\
& \left.\times\left. f(y) d y\right|^{2} \frac{d t}{t^{3}}\right)^{1 / 2} \\
& \leq C\left(\int_{\rho(x)}^{\infty} \mid \int_{\rho(x)<|x-y| \leq t} \frac{(1+|x-y| / \rho(x))^{-1}}{|x-y|^{d-1}}\right. \\
& \left.\times\left.|(b(x)-b(y)) f(y)| d y\right|^{2} \frac{d t}{t^{3}}\right)^{1 / 2} \\
& \leq C \rho(x)\left(\int_{\rho(x)}^{\infty} \mid \sum_{k=1}^{\left[\log _{2} t / \rho(x)\right]+1} \frac{1}{\left(2^{k} \rho(x)\right)^{n}}\right. \\
& \times \int_{|x-y| \leq 2^{k} \rho(x)} \mid(b(x)-b(y)) \\
& \left.\times f(y)|d y|^{2} \frac{d t}{t^{3}}\right)^{1 / 2} \\
& \leq C \rho(x) M_{V, \eta}^{b} f(x) \\
& \times\left(\int_{\rho(x)}^{\infty}\left(\left[\frac{\log _{2} t}{\rho(x)}\right]+1\right)^{2} \frac{d t}{t^{3}}\right)^{1 / 2} \\
& \leq C M_{V, \eta}^{b} f(x) \text {. }
\end{aligned}
$$

Therefore, the estimates for $\left[b, \mu_{j}^{L}\right]_{\text {glob }}$ follow from those for $M_{V, \eta}^{b} f(x)$ by Lemmas 16 and 18 .

To deal with $\left[b, \mu_{j}^{L}\right]_{\text {loc }}$ we write

$$
\begin{aligned}
& {\left[b, \mu_{j}^{L}\right]_{\mathrm{loc}}(f)(x)} \\
& \quad \leq \mathrm{I}(x)+\left[b, \mu_{j}^{\Delta}\right]_{\mathrm{loc}}(f)(x)+\mathrm{II}(x),
\end{aligned}
$$

where $\left[b, \mu_{j}^{\Delta}\right]_{\text {loc }}(f)(x)$ is defined in Lemma 15 and

$$
\begin{aligned}
& \mathrm{I}(x)=\left(\int_{0}^{\rho(x)} \mid \int_{|x-y| \leq t}\left[K_{j}^{L}(x, y)-K_{j}^{\Delta}(x, y)\right]\right. \\
&\left.\times\left.(b(x)-b(y)) f(y) d y\right|^{2} \frac{d t}{t^{3}}\right)^{1 / 2},
\end{aligned}
$$

$$
\begin{aligned}
\mathrm{II}(x) \leq\left(\int_{\rho(x)}^{\infty} \mid \int_{|x-y| \leq \rho(x)} K_{j}^{L}(x, y)\right. & \\
& \left.\quad \times\left.(b(x)-b(y)) f(y) d y\right|^{2} \frac{d t}{t^{3}}\right)^{1 / 2} .
\end{aligned}
$$

For II $(x)$, by Lemma 8 with $N=0$,

II $(x)$

$$
\begin{aligned}
& \leq C\left(\int_{\rho(x)}^{\infty} \mid \int_{|x-y| \leq \rho(x)} \frac{1}{|x-y|^{d-1}}\right. \\
& \left.\times\left.|(b(x)-b(y)) f(y)| d y\right|^{2} \frac{d t}{t^{3}}\right)^{1 / 2} \\
& \leq C\left(\int_{\rho(x)}^{\infty} \mid \sum_{k=-\infty}^{0} \frac{1}{\left(2^{k-1} \rho(x)\right)^{d-1}}\right. \\
& \left.\times\left.\int_{|x-y| \leq 2^{k} \rho(x)}|(b(x)-b(y)) f(y)| d y\right|^{2} \frac{d t}{t^{3}}\right)^{1 / 2} \\
& \leq C\left(\int_{\rho(x)}^{\infty} \mid \sum_{k=-\infty}^{0} \frac{2^{k} \rho(x)}{\left(2^{k} \rho(x)\right)^{d}}\right. \\
& \left.\times\left.\int_{|x-y| \leq 2^{k} \rho(x)}|(b(x)-b(y)) f(y)| d y\right|^{2} \frac{d t}{t^{3}}\right)^{1 / 2} \\
& \leq C \rho(x) M_{V, \eta}^{b} f(x)\left(\int_{\rho(x)}^{\infty} \frac{d t}{t^{3}}\right)^{1 / 2} \\
& \leq C M_{V, \eta}^{b} f(x) .
\end{aligned}
$$

For I $(x)$, using Lemma 8 again, we have

$\mathrm{I}(x)$

$$
\begin{array}{r}
\leq C\left(\int_{0}^{\rho(x)} \mid \int_{|x-y| \leq t} \frac{1}{|x-y|^{d-1}}\left(\frac{|x-y|}{\rho(x)}\right)^{\sigma}\right. \\
\left.\quad \times\left.|(b(x)-b(y)) f(y)| d y\right|^{2} \frac{d t}{t^{3}}\right)^{1 / 2} \\
\leq C \rho(x)^{-\sigma}\left(\int_{0}^{\rho(x)} \mid \sum_{k=-\infty}^{0} \frac{1}{\left(2^{k-1} t\right)^{d-\sigma-1}}\right. \\
\quad \times \int_{|x-y| \leq 2^{k} t} \mid(b(x)-b(y)) \\
\left.\times f(y)|d y|^{2} \frac{d t}{t^{3}}\right)^{1 / 2}
\end{array}
$$




$$
\begin{aligned}
& \leq C \rho(x)^{-\sigma}\left(\int_{0}^{\rho(x)} \mid \sum_{k=-\infty}^{0} \frac{\left(2^{k}\right)^{\sigma+1} t^{\sigma+1}}{\left(2^{k} t\right)^{d}}\right. \\
& \quad \times \int_{|x-y| \leq 2^{k} t} \mid(b(x)-b(y)) \\
& \left.\times f(y)|d y|^{2} \frac{d t}{t^{3}}\right)^{1 / 2} \\
& \leq C \rho(x)^{-\sigma}\left(\int_{0}^{\rho(x)}\left|\sum_{k=-\infty}^{0}\left(2^{k}\right)^{\sigma+1} t^{\sigma+1}\right|^{2} \frac{d t}{t^{3}}\right)^{1 / 2} M_{V, \eta}^{b} f(x) \\
& \leq C \rho(x)^{-\sigma}\left(\int_{0}^{\rho(x)} t^{2 \sigma-1} d t\right)^{1 / 2} M_{V, \eta}^{b} f(x) \\
& \leq C M_{V, \eta}^{b} f(x) .
\end{aligned}
$$

Using Lemmas 15, 16, 17, and 18 and Proposition 12, by adapting an argument in [2], we can obtain the desired result.

\section{Conflict of Interests}

The authors declare that there is no conflict of interests regarding the publication of this paper.

\section{Acknowledgments}

The authors would like to express their hearty thanks to the referee's comments. This paper is supported by the National Natural Science Foundation of China (10961015, 11261023, and 11326092) and the Jiangxi Natural Science Foundation of China (20122BAB201011), GJJ12203.

\section{References}

[1] E. M. Stein, "On the functions of Littlewood-Paley, LUSin, and Marcinkiewicz," Transactions of the American Mathematical Society, vol. 88, pp. 430-466, 1958.

[2] W. Gao and L. Tang, "Boundedness for Marcinkiewicz integrals associated with Schrödinger operators," ProceedingsMathematical Sciences, vol. 124, no. 2, pp. 193-203, 2014.

[3] D. Chen and D. Zou, "The boundedness of Marcinkiewicz integral associated with Schrödinger operator and its commutator," Journal of Function Spaces, vol. 2014, Article ID 402713, 10 pages, 2014.

[4] L. Tang, "Weighted norm inequalities for commutators of Littlewood-Paley functions related to Schrödinger operators," Archive der Mathematik, vol. 102, pp. 215-236, 2014.

[5] Z. W. Shen, " $L^{p}$ estimates for Schrödinger operators with certain potentials," Annales de l'institut Fourier, vol. 45, no. 2, pp. 513546, 1995.

[6] J. Dziubański and J. Zienkiewicz, "Hardy space $H^{1}$ associated to Schrödinger operator with potential satisfying reverse Hölder inequality," Revista Matemática Iberoamericana, vol. 15, no. 2, pp. 279-296, 1999.

[7] L. Tang and J. Dong, "Boundedness for some Schrödinger type operators on Morrey spaces related to certain nonnegative potentials," Journal of Mathematical Analysis and Applications, vol. 355, no. 1, pp. 101-109, 2009.

[8] H. Lin and D. Yang, "Equivalent boundedness of Marcinkiewicz integrals on non-homogeneous metric measure spaces," Science China. Mathematics, vol. 57, no. 1, pp. 123-144, 2014.

[9] Y. Liang, D. Yang, and S. Yang, "Applications of Orlicz-Hardy spaces associated with operators satisfying Poisson estimates," Science China. Mathematics, vol. 54, no. 11, pp. 2395-2426, 2011.

[10] D. Yang and S. Yang, "Musielak-Orlicz-Hardy spaces associated with operators and their applications," Journal of Geometric Analysis, vol. 24, no. 1, pp. 495-570, 2014.

[11] D. Yang and Y. Zhou, "Localized Hardy spaces $H^{1}$ related to admissible functions on RD-spaces and applications to Schrödinger operators," Transactions of the American Mathematical Society, vol. 363, no. 3, pp. 1197-1239, 2011.

[12] D. Yang and Y. Zhou, "Localized Morrey-Campanato spaces on metric measure spaces and applications to Schrödinger operators," Nagoya Mathematical Journal, vol. 198, pp. 77-119, 2010.

[13] Y. Dachun, Y. Dongyong, and Y. Zhou, "Localized BMO and BLO spaces on RD-spaces and applications to Schrödinger operators," Communications on Pure and Applied Analysis, vol. 9, no. 3, pp. 779-812, 2010.

[14] B. Bongioanni, E. Harboure, and O. Salinas, "Commutators of Riesz transforms related to Schrödinger operators," The Journal of Fourier Analysis and Applications, vol. 17, no. 1, pp. 115-134, 2011.

[15] L. Tang, "Weighted norm inequalities for Schrödinger type operators," Forum Mathematicum, 2013.

[16] B. Bongioanni, E. Harboure, and O. Salinas, "Class of weights related to Schrödinger operators," Journal of Mathematical Analysis and Applications, vol. 373, no. 2, pp. 563-579, 2011. 


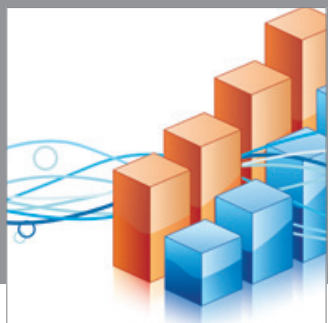

Advances in

Operations Research

mansans

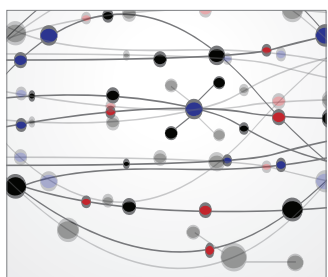

The Scientific World Journal
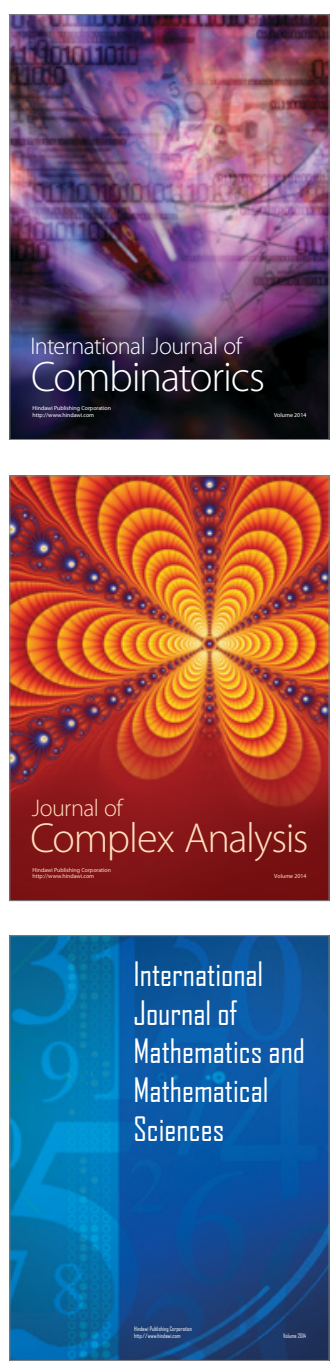
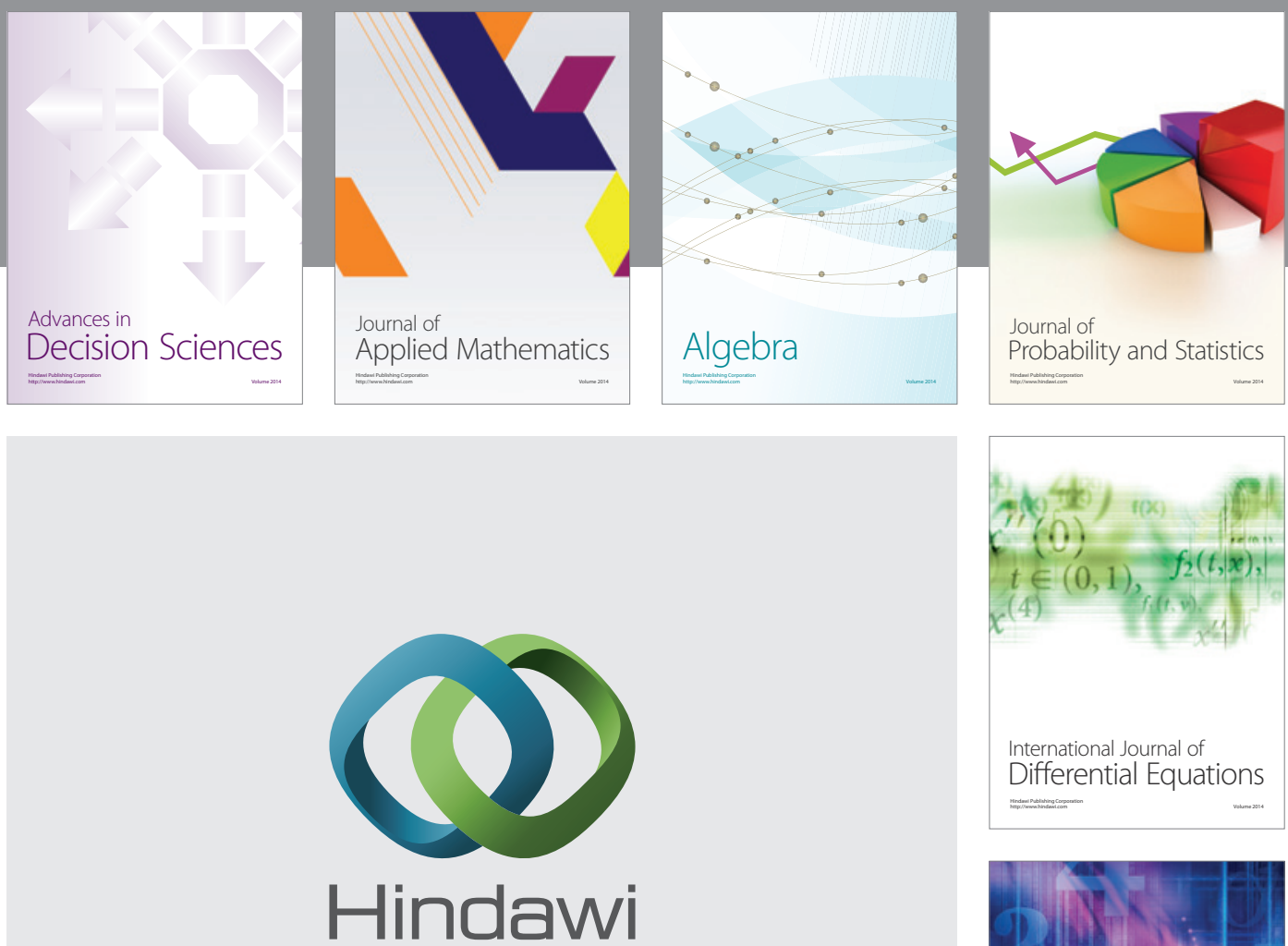

Submit your manuscripts at http://www.hindawi.com
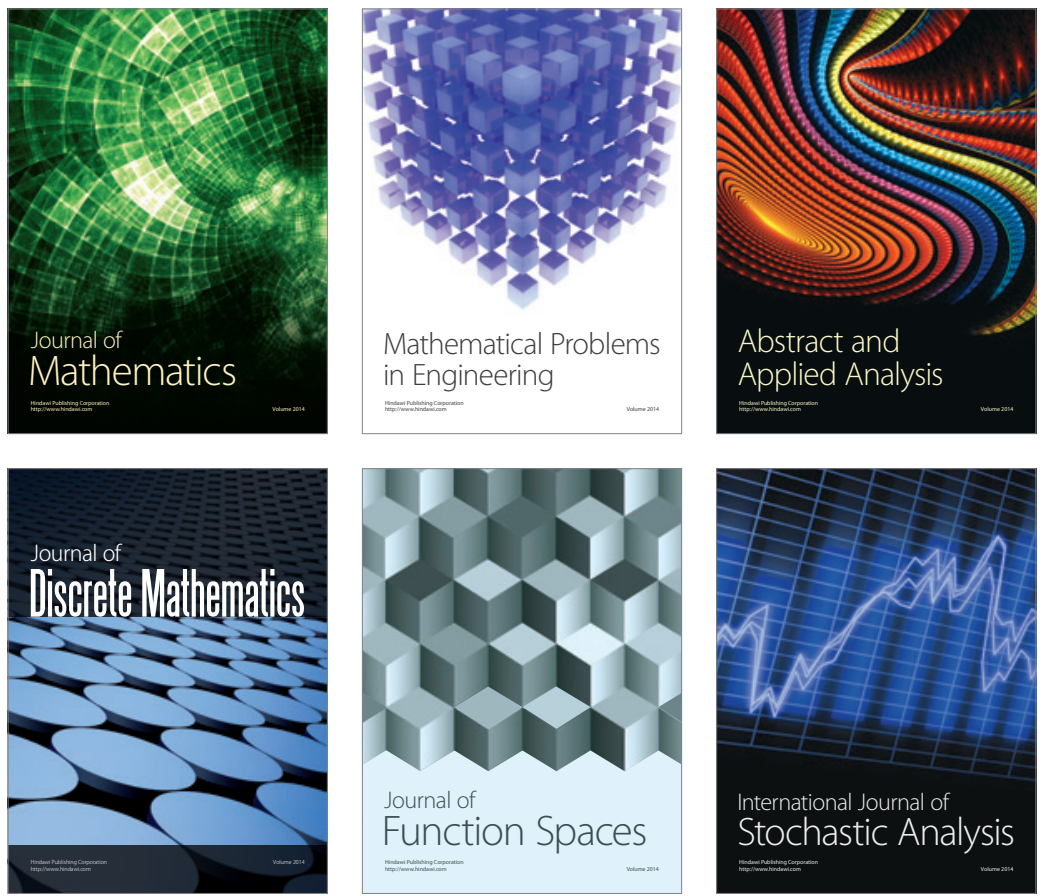

Journal of

Function Spaces

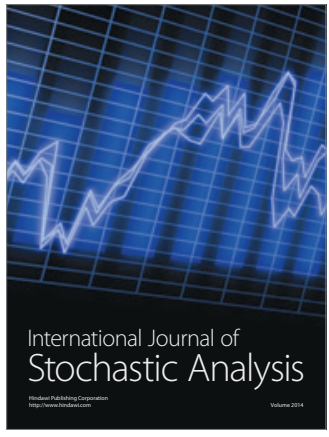

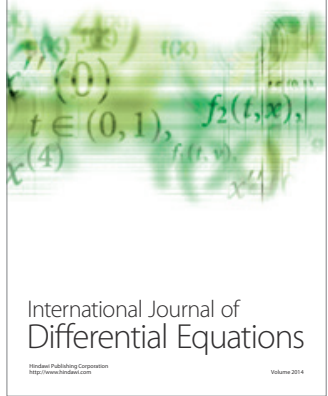
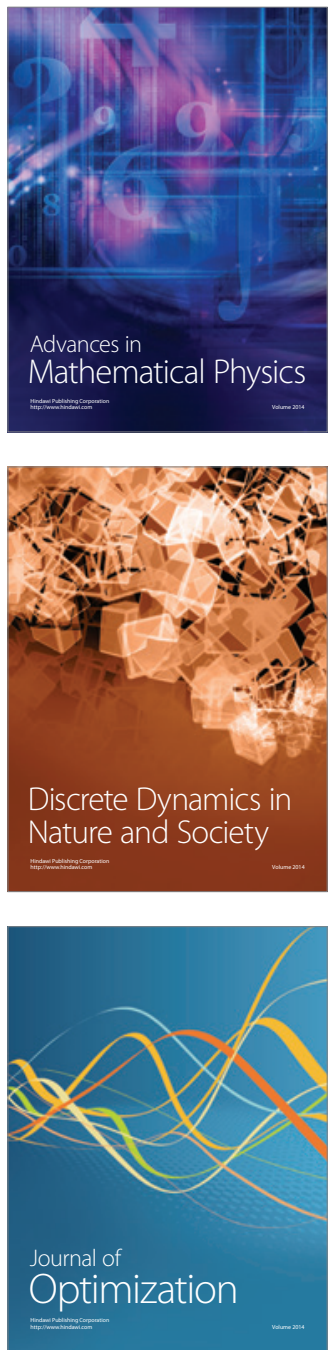